\title{
Whirling orbits around twirling black holes from conformal symmetry
}

\author{
Shahar $\operatorname{Hadar}^{a}$ and Achilleas P. Porfyriadis ${ }^{b}$ \\ ${ }^{a}$ Department of Applied Mathematics and Theoretical Physics, University of Cambridge, \\ Wilberforce Road, Cambridge CB3 OWA, U.K. \\ ${ }^{b}$ Department of Physics, UCSB, \\ Santa Barbara, CA 93106, U.S.A. \\ E-mail: shaharhadar@gmail.com, app@physics.ucsb.edu
}

ABSTRACT: Dynamics in the throat of rapidly rotating Kerr black holes is governed by an emergent near-horizon conformal symmetry. The throat contains unstable circular orbits at radii extending from the ISCO down to the light ring. We show that they are related by conformal transformations to physical plunges and osculating trajectories. These orbits have angular momentum arbitrarily higher than that of ISCO. Using the conformal symmetry we compute analytically the radiation produced by the physical orbits. We also present a simple formula for the full self-force on such trajectories in terms of the self-force on circular orbits.

KeYwords: Black Holes, Space-Time Symmetries, AdS-CFT Correspondence

ARXIV EPRINT: 1611.09834 


\section{Contents}

1 Introduction 1

2 Trajectories \& mapping 2

3 Radiation field $\quad 4$

3.1 Physical orbits 6

$\begin{array}{llr}4 & \text { Self-force } & 7\end{array}$

$\begin{array}{lr}\text { A Conformal mappings of near-NHEK } & 8\end{array}$

\section{Introduction}

Recently [1] gravitational waves from a binary black hole $(\mathrm{BH})$ coalescence were directly observed for the first time by LIGO [2]. This remarkable achievement is an important milestone in gravitational physics. It marks the beginning of a period in which progressively accurate signatures of increasingly numerous strong-gravity events from across the universe will be collected and analysed. The LISA mission [3], expected to be launched in 2028, will allow accurate measurement of gravitational waves from extreme-mass-ratio-inspirals (EMRI) of compact stellar-size objects into supermassive BHs. Specifically, LISA is expected to provide waveforms from the late, strong gravity dominated, stages of the EMRI that will probe the spacetime geometry in the vicinity of the horizon and precision-test $\mathrm{BH}$ uniqueness.

It is believed that a significant fraction of the supermassive BHs residing at the centers of galaxies are rapidly rotating $[4,5]$. Several of them are thought to be spinning within $98 \%$ of extremality. The enhanced symmetries of such near-extremal Kerr BHs were first studied in the context of quantum gravity: understanding of the near-horizon near-extremal limit of Kerr BHs in [6] motivated the Kerr/CFT conjecture [7] which asserts that quantum gravity in the near-horizon region of near-extreme Kerr is holographically dual to a $1+1$ dimensional conformal field theory. However, these enhanced symmetries govern any physical process near such extreme BHs, including those which are potentially observable up in the sky. In the astrophysical context, there is a growing number of applications in which nearextremal near-horizon symmetries are utilized to study realistic processes in the vicinity of such BHs. Applications include gravitational waves [8-12], magnetospheres [13-17], and, recently, electromagnetic emissions [18]; see also [19, 20].

In this paper we extend the analyses of gravitational wave signals from near-extremal Kerr EMRIs performed in [8-11]. Refs. [8-11] studied circular and plunging orbits with angular momentum equal to that of the innermost-stable-circular-orbit (ISCO). In this 
paper we consider orbits with angular momentum arbitrarily higher than that of ISCO. ${ }^{1}$ We first solve for a 1-parameter family of unstable equatorial circular orbits, labeled by their angular momenta, whose radii extend from the ISCO down to the light ring. We then find a 1-parameter family of conformal mappings that transform them, altogether, to a 2-parameter family of physical orbits, labeled by their energy and angular momentum. These are either plunges or grazing "zoom-whirl" orbits. They include plunges that naively (i.e. neglecting backreaction) overspin the $\mathrm{BH}$ beyond extremality [21-23]. For all physical orbits we solve for the corresponding field profile including the observed waveform at future null infinity. Finally, we discuss an application of our results to the study of the self-force, arguing that the self-force on any of the 2-parameter family of physical orbits may also be obtained via the above-mentioned coordinate transformation from the much simpler case of the circular orbit.

The rest of the paper is organized as follows. In section 2 we introduce a family of transformations that map unstable circular orbits in the near-horizon geometry of nearextreme Kerr to generic 2-parameter orbits with angular momentum higher than that of ISCO. In section 3 we use these mappings to solve for the field emitted by such orbits, including the observed radiation at future null infinity. In section 4 we argue that the full self-force for the generic orbits - not only the radiative part - is given via our mappings from the simpler circular orbit case by a compact analytic formula. The appendix contains details of the derivation of the conformal mappings employed in this paper.

\section{Trajectories \& mapping}

In Boyer-Lindquist coordinates, the Kerr metric describing a $\mathrm{BH}$ with mass $M$ and angular momentum $J=a M$ is given by $(G=c=1)$

$$
d s^{2}=-\frac{\Delta}{\hat{\rho}^{2}}\left(d \hat{t}-a \sin ^{2} \theta d \hat{\phi}\right)^{2}+\frac{\sin ^{2} \theta}{\hat{\rho}^{2}}\left(\left(\hat{r}^{2}+a^{2}\right) d \hat{\phi}-a d \hat{t}\right)^{2}+\frac{\hat{\rho}^{2}}{\Delta} d \hat{r}^{2}+\hat{\rho}^{2} d \theta^{2},
$$

where

$$
\Delta=\hat{r}^{2}-2 M \hat{r}+a^{2}, \quad \hat{\rho}^{2}=\hat{r}^{2}+a^{2} \cos ^{2} \theta .
$$

The horizons are at $r_{ \pm}=M \pm \sqrt{M^{2}-a^{2}}$. The angular momentum of Kerr BHs is bounded from above by the extremal value $a=M$. Close to extremality the near-horizon dynamics is greatly simplified by the presence of conformal symmetry [6, 7]. The limit is best explored in Bardeen-Horowitz coordinates

$$
R=\frac{\hat{r}-r_{+}}{r_{+}}, \quad T=\frac{\hat{t}}{2 M}, \quad \Phi=\hat{\phi}-\frac{\hat{t}}{2 M} .
$$

Parameterizing the deviation from extremality by

$$
\kappa=\sqrt{1-a^{2} / M^{2}} \ll 1
$$

\footnotetext{
${ }^{1}$ Our analysis is carried out for a massless scalar field, but it is readily generalizable to electromagnetism and gravity along the lines of [9].
} 
the near-horizon metric at $R \sim \kappa$ reads

$$
d s^{2}=2 M^{2} \Gamma(\theta)\left[-R(R+2 \kappa) d T^{2}+\frac{d R^{2}}{R(R+2 \kappa)}+d \theta^{2}+\Lambda(\theta)^{2}(d \Phi+(R+\kappa) d T)^{2}\right],
$$

where

$$
\Gamma(\theta)=\frac{1+\cos ^{2} \theta}{2}, \quad \Lambda(\theta)=\frac{2 \sin \theta}{1+\cos ^{2} \theta} .
$$

This metric, which describes the near-horizon geometry of near-extreme Kerr, is referred to as near-NHEK [24] and it solves the Einstein equation on its own. The near-horizon geometry of extreme Kerr, referred to as NHEK, is given by [6]

$$
d s^{2}=2 M^{2} \Gamma(\theta)\left[-\tilde{R}^{2} d \tilde{T}^{2}+\frac{d \tilde{R}^{2}}{\tilde{R}^{2}}+d \theta^{2}+\Lambda(\theta)^{2}(d \tilde{\Phi}+\tilde{R} d \tilde{T})^{2}\right] .
$$

This metric solves the Einstein equation on its own as well. Note that NHEK also describes the near-horizon geometry at $\kappa \ll R \ll 1$ in the throat of near-extreme Kerr (see e.g. appendix $\mathrm{A}$ in [11]). NHEK and near-NHEK are different patches in the global-NHEK space-time $[6]$

$$
d s^{2}=2 M^{2} \Gamma(\theta)\left[-\left(1+\rho^{2}\right) d \tau^{2}+\frac{d \rho^{2}}{1+\rho^{2}}+d \theta^{2}+\Lambda(\theta)^{2}(d \varphi+\rho d \tau)^{2}\right] .
$$

The isometry group is $\mathrm{SL}(2, \mathbb{R}) \times \mathrm{U}(1)$ with time translations being part of the $\mathrm{SL}(2, \mathbb{R})$.

In the near-NHEK geometry (2.5) there exist equatorial circular orbits at any radius above the light ring

$$
R_{0} \geq\left(\frac{2}{\sqrt{3}}-1\right) \kappa
$$

Their trajectories are given by

$$
\begin{aligned}
& R=R_{0}, \\
& \Phi=\Phi_{0}-\frac{3\left(R_{0}+\kappa\right)}{4} T,
\end{aligned}
$$

and they carry near-NHEK energy and angular momentum

$$
\begin{aligned}
& E=-\frac{2 M \kappa^{2}}{\sqrt{3 R_{0}^{2}+6 R_{0} \kappa-\kappa^{2}}}, \\
& L=\frac{2 M\left(R_{0}+\kappa\right)}{\sqrt{3 R_{0}^{2}+6 R_{0} \kappa-\kappa^{2}}} .
\end{aligned}
$$

These circular orbits are all unstable (see e.g. appendix B in [9]). Therefore, naively, one might think that they are irrelevant for realistic physical situations. This is not the case. Consider the following conformal transformations

$$
\begin{aligned}
& R=\sqrt{r(r+2 \kappa)}(\sinh \kappa t+\chi \cosh \kappa t)-\chi(r+\kappa)-\kappa, \\
& T=\frac{1}{\kappa} \ln \frac{\sqrt{r(r+2 \kappa)} \cosh \kappa t-(r+\kappa)}{\sqrt{R(R+2 \kappa)}}, \\
& \Phi=\phi-\frac{1}{2} \ln \frac{\sqrt{r(r+2 \kappa)}-(r+\kappa) \cosh \kappa t+\kappa \sinh \kappa t}{\sqrt{r(r+2 \kappa)}-(r+\kappa) \cosh \kappa t-\kappa \sinh \kappa t} \frac{R+2 \kappa}{R},
\end{aligned}
$$


where $\chi$ is a constant. As explained in the appendix, these transformations may be thought of as a $\tilde{T} \rightarrow \tilde{T}-\chi$ translation followed by a $\tau \rightarrow \tau-\pi / 2$ translation. The transformations (2.13) leave the near-NHEK metric invariant,

$$
d s^{2}=2 M^{2} \Gamma(\theta)\left[-r(r+2 \kappa) d t^{2}+\frac{d r^{2}}{r(r+2 \kappa)}+d \theta^{2}+\Lambda(\theta)^{2}(d \phi+(r+\kappa) d t)^{2}\right],
$$

but map the trajectory $(2.10)$ to

$$
\begin{aligned}
t(r)= & \frac{1}{\kappa} \ln \frac{R_{0}+\kappa+\chi(r+\kappa)+D}{(\chi+1) \sqrt{r(r+2 \kappa)}} \\
\phi(r)= & \Phi_{0}-\frac{3\left(R_{0}+\kappa\right)}{4 \kappa} \ln \frac{r+\kappa+\chi\left(R_{0}+\kappa\right)-D}{\left(\chi^{2}-1\right) \sqrt{R_{0}\left(R_{0}+2 \kappa\right)}}+\frac{1}{2} \ln \frac{\left(R_{0}+2 \kappa\right) r}{R_{0}(r+2 \kappa)} \\
& +\frac{1}{2} \ln \frac{r^{2}+R_{0}^{2}+(\chi-1) R_{0} r+(\chi+1)^{2} \kappa^{2}-\left(r-R_{0}\right) D-\kappa(\chi+1)(D-2 r)}{r^{2}+R_{0}^{2}+(\chi-1) R_{0} r+(\chi+1)^{2} \kappa^{2}-\left(r-R_{0}\right) D+\kappa(\chi+1)\left(D+2 R_{0}\right)},
\end{aligned}
$$

where

$$
D=\sqrt{r^{2}+2 r\left(\kappa+\chi\left(R_{0}+\kappa\right)\right)+\left(\kappa(\chi+1)+R_{0}\right)^{2}} .
$$

These are a 2-parameter family of near-NHEK trajectories which carry near-NHEK energy and angular momentum ${ }^{2}$

$$
\begin{aligned}
& e=\frac{2 M \kappa^{2} \chi}{\sqrt{3 R_{0}^{2}+6 R_{0} \kappa-\kappa^{2}}}, \\
& l=\frac{2 M\left(R_{0}+\kappa\right)}{\sqrt{3 R_{0}^{2}+6 R_{0} \kappa-\kappa^{2}}} .
\end{aligned}
$$

For $\chi>-1$ the trajectories (2.15) are plunges which start from the near-NHEK boundary at $t=0$ and fall into the future horizon. For $\chi<-1$ they are osculating orbits which penetrate the throat at the near-NHEK boundary at $t=0$ and then exit it at some later finite time. This is illustrated in figure 1.

\section{Radiation field}

Consider a point-like scalar-charged object on a geodesic $x_{*}(\tau)$ which couples to a massless scalar field $\Psi$. The system is described by the action

$$
S=-\frac{1}{2} \int d^{4} x \sqrt{-g}\left[(\partial \Psi)^{2}+8 \pi \lambda \Psi \mathcal{S}\right]
$$

where $\lambda$ is a coupling constant and

$$
\mathcal{S}=-\int d \tau(-g)^{-1 / 2} \delta^{4}\left(x-x_{*}(\tau)\right)
$$

\footnotetext{
${ }^{2}$ Note that while $l$ coincides with the asymptotic angular momentum $\hat{L}$, the near-NHEK energy $e$ measures the difference $\hat{E}-\hat{L} /(2 M)$.
} 


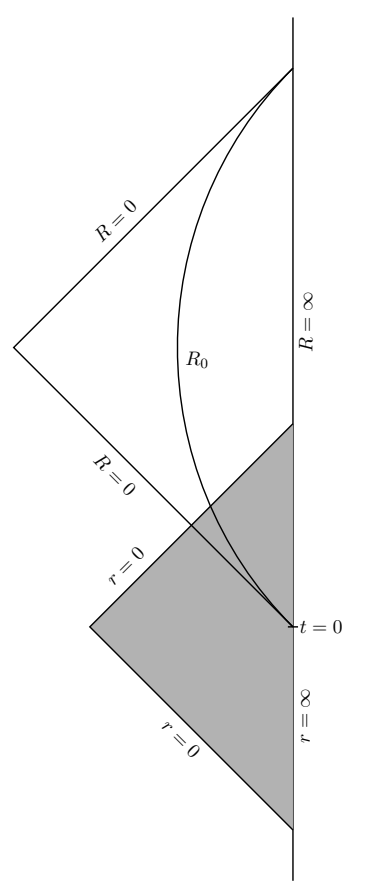

(a) $\chi>0$.

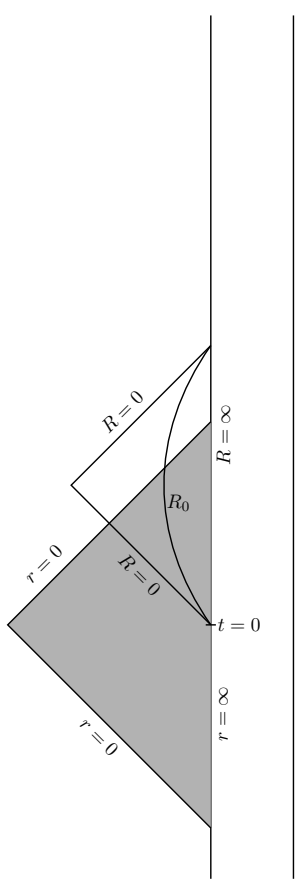

(b) $-1<\chi<0$.

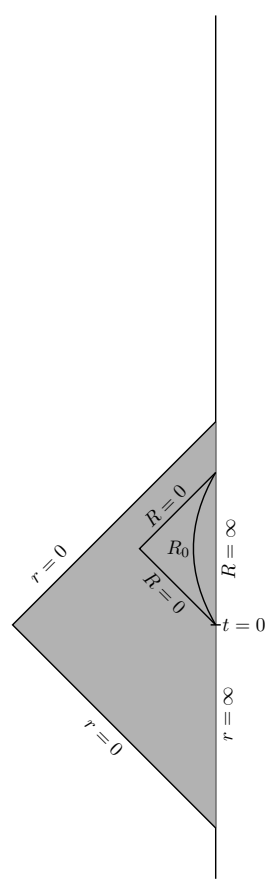

(c) $\chi<-1$.

Figure 1. Penrose diagrams of the throat geometry for different values of $\chi$. In each case the asymptotically flat region of Kerr is to be attached to the shaded wedge. The wedge bounded by $R=0$ and $R=\infty$ is a near-NHEK patch in coordinates (2.5). The wedge bounded by $r=0$ and $r=\infty$ is a near-NHEK patch in coordinates (2.14). The line at $R=R_{0}$ is a circular orbit in (2.5) which in (2.14) is seen as a plunging orbit in (a) and (b), or as an osculating orbit in (c).

We are interested in solving for the radiation field produced by an object on the physical orbits (2.15). We may do so analytically by solving first the much simpler case corresponding to the circular orbits (2.10) and then transforming the solution according to (2.13).

For the circular orbits (2.10) in the near-NHEK patch (2.5), decomposing the scalar field as

$$
\Psi=\sum_{\ell, m} e^{i m(\Phi-\Omega T)} S_{\ell}(\theta) \mathcal{R}_{\ell m}(R)
$$

with

$$
\Omega=-\frac{3\left(R_{0}+\kappa\right)}{4}
$$

and substituting into the equations of motion for (3.1) one finds

$$
\begin{aligned}
R(R+2 \kappa) \mathcal{R}^{\prime \prime}+2(R+\kappa) \mathcal{R}^{\prime}+\left[\frac{m^{2}(R+\kappa+\Omega)^{2}}{R(R+2 \kappa)}+m^{2}-K\right] \mathcal{R} & =Q R_{0} \delta\left(R-R_{0}\right), \\
& {\left[\frac{1}{\sin \theta} \partial_{\theta}\left(\sin \theta \partial_{\theta}\right)+K-\frac{m^{2}}{\sin ^{2} \theta}-\frac{m^{2}}{4} \sin ^{2} \theta\right] S_{\ell}=0 }
\end{aligned}
$$

where $Q=-\frac{\sqrt{3} \lambda}{2 M} S_{\ell}(\pi / 2)$. The solutions $S(\theta)$ of the (extremal) spheroidal wave equation (3.5) and their associated eigenvalues $K$ are well known (e.g. they are built in Mathematica as SpheroidalPS and SpheroidalEigenvalue). The solution to (3.4) is a Green's 
function constructed from homogeneous solutions properly matched at $R=R_{0}$. A particularly useful basis of homogeneous solutions for near-NHEK physics is [11]

$$
\begin{aligned}
& \mathcal{R}_{\text {in }}=R^{-\frac{i m}{2}(\Omega / \kappa+1)}\left(\frac{R}{2 \kappa}+1\right){ }_{2}^{\frac{i m}{2}(\Omega / \kappa-1)} F_{1}\left(h-i m, 1-h-i m ; 1-i m(\Omega / \kappa+1) ;-\frac{R}{2 \kappa}\right), \\
& \mathcal{R}_{N}=R^{-h}\left(\frac{2 \kappa}{R}+1\right)_{2}^{\frac{i m}{2}(\Omega / \kappa-1)} F_{1}\left(h-i m, h+i m \Omega / \kappa ; 2 h ;-\frac{2 \kappa}{R}\right),
\end{aligned}
$$

where

$$
h=\frac{1}{2}+\sqrt{K-2 m^{2}+\frac{1}{4}} .
$$

$\mathcal{R}_{\text {in }}$ obeys ingoing boundary conditions at the horizon, $\mathcal{R}_{\text {in }}(R \rightarrow 0)=R^{-\frac{i m}{2}(\Omega / \kappa+1)}$, and $\mathcal{R}_{N}$ obeys Neumann boundary conditions at the near-NHEK boundary, $\mathcal{R}_{N}(R \gg \kappa)=R^{-h}$. The solution to equation (3.4) with these boundary conditions is given by

$$
\mathcal{R}_{c}=\frac{Q R_{0}}{A(1-2 h)} \mathcal{R}_{\mathrm{in}}\left(R_{<}\right) \mathcal{R}_{N}\left(R_{>}\right)
$$

where $R_{<}$and $R_{>}$are the lesser and greater of $R_{0}$ and $R$, respectively, and

$$
A=\frac{\Gamma(2 h-1) \Gamma(1-i m(\Omega / \kappa+1))}{\Gamma(h-i m) \Gamma(h-i m \Omega / \kappa)}(2 \kappa)^{1-h-i m(\Omega / \kappa+1) / 2} .
$$

\subsection{Physical orbits}

The solution for the physical orbits (2.15) in the near-NHEK patch (2.14) is obtained analytically by applying the transformation (2.13) on the above solution for the circular orbit. This yields the near-NHEK solution with causal boundary conditions at the horizon $r=0$ and Neumann at $r \gg \kappa$. Indeed, for $r \gg \kappa$ and fixed $t, \phi$ the transformation (2.13) reduces to

$$
\begin{aligned}
& R \approx r[\sinh \kappa t+\chi(\cosh \kappa t-1)] \gg \kappa \\
& T \approx-\frac{1}{\kappa} \ln \left(\operatorname{coth} \frac{\kappa t}{2}+\chi\right) \\
& \Phi \approx \phi
\end{aligned}
$$

so that plugging into (3.8) and (3.3) we obtain, for $t>0$,

$$
\Psi(r \gg \kappa)=\frac{1}{\sqrt{2 \pi}} \int d \omega \sum_{\ell m} \frac{Q R_{0} \mathcal{R}_{\mathrm{in}}\left(R_{0}\right)}{\kappa A(1-2 h)} \mathcal{N} e^{i(m \phi-\omega t)} S_{\ell}(\theta) r^{-h},
$$

where

$$
\mathcal{N}=\frac{1}{\sqrt{2 \pi}} \int_{0}^{\infty} d y e^{i \frac{\omega}{\kappa} y}\left(\operatorname{coth} \frac{y}{2}+\chi\right)^{-\frac{3 i m\left(R_{0}+\kappa\right)}{4 \kappa}}[\sinh y+\chi(\cosh y-1)]^{-h}
$$

In order to find the radiation field at asymptotically flat infinity one needs to match this near-NHEK solution to a solution in the far Kerr geometry. This may be done using the method of matched asymptotic expansions (MAE). For a detailed presentation of the 
method applied to a different but similar solution in near-NHEK we refer the reader to section 4.4 in [9]. Here we will only review the general idea and necessary definitions and give the final result for the orbits (2.15) considered in this paper.

Consider a scalar field on the full near-extreme Kerr geometry in Boyer-Lindquist coordinates and expand in modes

$$
\Psi=\frac{1}{\sqrt{2 \pi}} \int d \hat{\omega} \sum_{\ell m} e^{i(m \hat{\phi}-\hat{\omega} \hat{t})} \hat{S}_{\ell}(\theta) \hat{\mathcal{R}}_{\ell m \hat{\omega}}(\hat{r})
$$

with $\hat{S}_{\ell}(\theta)$ the standard Kerr spheroidal harmonics. Identify the near-NHEK geometry which contains the orbits studied in this paper as the region given by $r=\left(\hat{r}-r_{+}\right) / r_{+} \ll 1$. Let the dimensionless Hawking temperature and rescaled near-superradiant frequency be

$$
\tau_{H}=\frac{r_{+}-r_{-}}{r_{+}}, \quad n=4 M \frac{\hat{\omega}-m \Omega_{H}}{\tau_{H}},
$$

with $\Omega_{H}=a /\left(2 M r_{+}\right)$the horizon angular velocity. ${ }^{3}$ The solution for the field in the far Kerr region, $r \gg \max \left(\tau_{H}, n \tau_{H}\right)$, with no incoming radiation from past null infinity is given by equation (4.14) in [9]. Identifying $\omega=(n-m) \kappa$ the near-NHEK solution may be matched to the far Kerr solution in the overlap region $\max \left(\tau_{H}, n \tau_{H}\right) \ll r \ll 1$. It should be noted that matching to the purely outgoing solution at null infinity requires modifying the Neumann boundary condition on the near-NHEK solution to so called "leaky" boundary conditions [8]. These are such that at the boundary of near-NHEK, which is in the matching region, we have the appropriate ratio of Dirichlet to Neumann modes that ensures the correct amount of radiation leaks through the near-NHEK boundary and reaches future null infinity. In the end, the result for the waveform at future null infinity, for $m>0$, is given by:

$$
\begin{aligned}
\hat{\mathcal{R}}_{\ell m \hat{\omega}}(\hat{r} \rightarrow \infty)=\frac{2 \sqrt{3} \lambda}{\pi}(-2 i \kappa)^{h-1} e^{\frac{\pi m}{2}} m^{h-1+i m}\left(\frac{R_{0}}{2 \kappa}\right)^{1-\frac{i m}{2}(\Omega / \kappa+1)}\left(\frac{R_{0}}{2 \kappa}+1\right)^{\frac{i m}{2}(\Omega / \kappa-1)} \times \\
\quad \times S_{\ell}(\pi / 2)_{2} F_{1}\left(h-i m, 1-h-i m ; 1-i m(\Omega / \kappa+1) ;-\frac{R_{0}}{2 \kappa}\right) \times \\
\quad \times \frac{\Gamma(1-2 h) \Gamma(h-i m \Omega / \kappa)}{\Gamma(2 h-1) \Gamma(1-i m(\Omega / \kappa+1))} \Gamma(h-i m)^{2} \sin (2 \pi h) \mathcal{N} \times \\
\quad \times\left[1-(-2 i m \kappa)^{2 h-1} \frac{\Gamma(1-2 h)^{2} \Gamma(h-i m)^{2}}{\Gamma(2 h-1)^{2} \Gamma(1-h-i m)^{2}} \frac{\Gamma(h-i(n-m))}{\Gamma(1-h-i(n-m))}\right]^{-1} r^{-1+i m} e^{i m r / 2}
\end{aligned}
$$

\section{Self-force}

In this section we propose another application of the near-extreme conformal symmetry: computation of the self-force (SF) on generic equatorial orbits in the near-horizon region. We deal with the scalar case, as in throughout this paper, but a generalization to gravity should be possible along lines similar to those taken in [9]. In the gravitational case, reconstruction of the metric is required to find the full SF (see [25] for recent progress).

\footnotetext{
${ }^{3}$ Note that to leading order $\tau_{H}=2 \kappa$.
} 
The scalar $\mathrm{SF}$ is given by

$$
F_{a}^{(S F)}=\nabla_{a} \Psi^{R}
$$

where $\Psi^{R}$ is the regular piece of the field as defined by Detweiler and Whiting [26]. The transformation (2.13) is a diffeomorphism: locally, it is just a change of coordinates. Since the singular-regular decomposition is defined locally and is insensitive to changes in the global spacetime structure, the same decomposition will hold after performing (2.13). Now, the quantity $F_{a}^{(S F)}$ transforms to the plunge coordinates as a proper vector:

$$
F_{a}^{\text {(plunge) }}=\frac{\partial X^{A}}{\partial x^{a}} F_{A}^{(\text {circular })},
$$

where $X^{A}$ stands for the coordinates in (2.5) and $x^{a}$ stands for the coordinates in (2.14). $X^{A}\left(x^{a}\right)$ is given by (2.13). This gives a remarkably simple formula for the $S F$ on generic near-horizon orbits from the circular SF.

It is important to note that, to leading order in the deviation from extremality, the SF computation is insensitive to the boundary conditions in the asymptotically flat region. ${ }^{4} \mathrm{It}$ is possible, therefore, to use the solution (3.8) for SF computations without needing to worry about matching as in (3.15). It will be interesting to numerically test our formula (4.2) for the self-force.

\section{Acknowledgments}

We are grateful to Leor Barack for useful conversations. SH is supported by the Blavatnik Postdoctoral Fellowship. APP is supported by NSF grant PHY-1504541.

\section{A Conformal mappings of near-NHEK}

In this appendix we give some details in relation to the conformal transformation (2.13) employed in this paper in order to map unstable circular orbits in near-NHEK to physical plunges and osculating orbits. In particular, we show how (2.13) may be obtained by composing a NHEK time translation $\tilde{T} \rightarrow \tilde{T}-\chi$ with a global time translation $\tau \rightarrow \tau-\pi / 2$.

First embed a near-NHEK patch,

$$
d s^{2}=2 M^{2} \Gamma(\theta)\left[-\tilde{r}(\tilde{r}+2 \kappa) d \tilde{t}^{2}+\frac{d \tilde{r}^{2}}{\tilde{r}(\tilde{r}+2 \kappa)}+d \theta^{2}+\Lambda(\theta)^{2}(d \tilde{\phi}+(\tilde{r}+\kappa) d \tilde{t})^{2}\right],
$$

inside a NHEK patch (2.7) as shown in figure 2. The transformation between the coordinates $(\tilde{T}, \tilde{R}, \tilde{\Phi})$ and $(\tilde{t}, \tilde{r}, \tilde{\phi})$ may be found in [9]. Following that up by translation $\tilde{T} \rightarrow$ $\tilde{T}-\chi$ one finds the following mapping between the near-NHEK patches (2.5) and (A.1).

$$
\begin{aligned}
R & =\tilde{r}+\chi e^{\kappa \tilde{t}} \sqrt{\tilde{r}(\tilde{r}+2 \kappa)}, \\
T & =\tilde{t}+\frac{1}{2 \kappa} \ln \frac{\tilde{r}(\tilde{r}+2 \kappa)}{R(R+2 \kappa)}, \\
\Phi & =\tilde{\phi}-\frac{1}{2} \ln \frac{(R+2 \kappa) \tilde{r}}{R(\tilde{r}+2 \kappa)} .
\end{aligned}
$$

\footnotetext{
${ }^{4}$ Apart from certain fine-tuned boundary conditions that eliminate the Neumann mode at the boundary.
} 


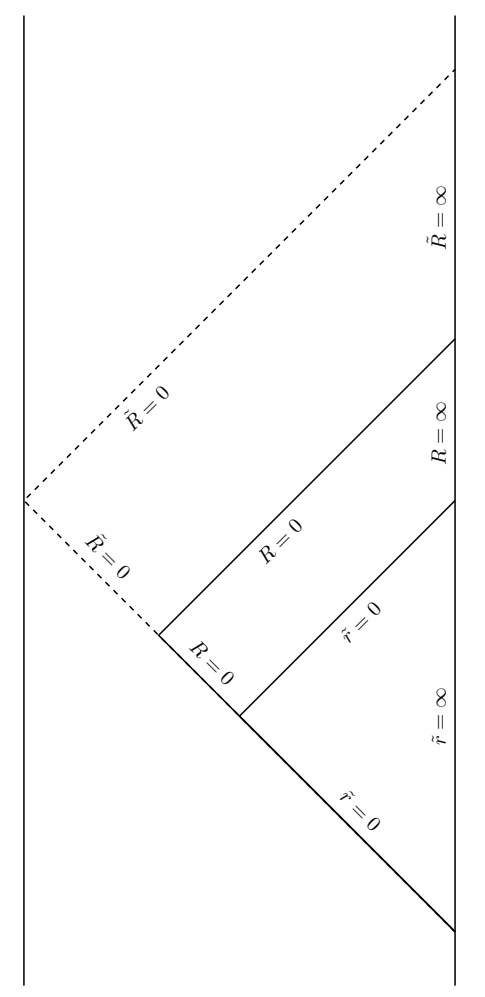

Figure 2. Penrose diagram illustrating the setup for deriving the transformation (A.2). The solid wedge bounded by $\tilde{r}=0$ and $\tilde{r}=\infty$ is near-NHEK. The solid wedge bounded by $R=0$ and $R=\infty$ is also near-NHEK. Both are embedded in the dashed NHEK wedge bounded by $\tilde{R}=0$ and $\tilde{R}=\infty$. The near-NHEK wedges are related to each other by a NHEK time translation $\tilde{T} \rightarrow \tilde{T}-\chi$.

Note that the transformation of the circular orbit (2.10) in (2.5) gives rise to orbits in (A.1) which are qualitatively very similar to the ones studied in the paper. The only difference is that these orbits enter the throat in the infinite near-NHEK past $(\tilde{t}=-\infty)$. In the terminology of [10] these are "slow" plunging or osculating orbits.

In this paper we considered the radiation from "fast" plunging or osculating orbits. These enter the throat at some finite near-NHEK time (which we set to $t=0$ ). Such "fast" orbits may be obtained by a transformation corresponding to a global time translation $\tau \rightarrow \tau-\pi / 2$, illustrated in figure 3. This mapping between the near-NHEK patches (A.1) and (2.14) is given by

$$
\begin{aligned}
& \tilde{r}=\sqrt{r(r+2 \kappa)} \sinh \kappa t-\kappa, \\
& \tilde{t}=\frac{1}{2 \kappa} \ln \frac{\sqrt{r(r+2 \kappa)} \cosh \kappa t-(r+\kappa)}{\sqrt{r(r+2 \kappa)} \cosh \kappa t+(r+\kappa)}, \\
& \tilde{\phi}=\phi+\frac{1}{2} \ln \frac{(r+\kappa) \sinh \kappa t+\kappa \cosh \kappa t}{(r+\kappa) \sinh \kappa t-\kappa \cosh \kappa t} .
\end{aligned}
$$

Note that the transformation (A.3) corresponds to the $\chi=0$ case of (2.13).

The transformation (2.13) is a composition of (A.2) with (A.3). 


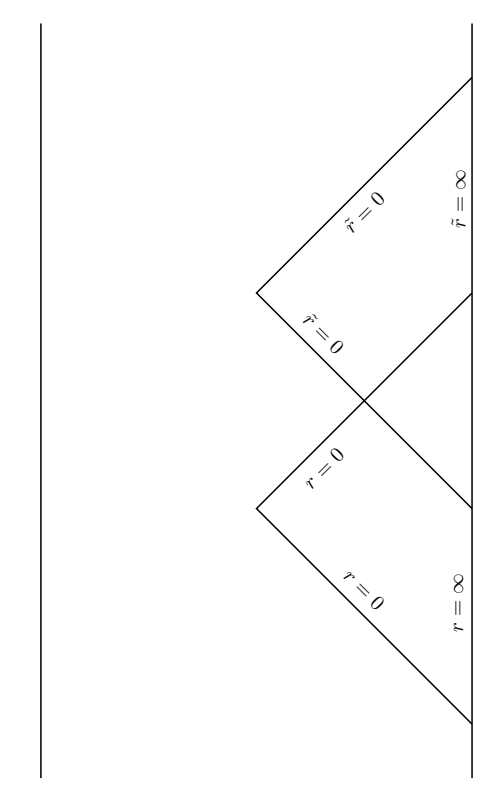

Figure 3. Penrose diagram illustrating the setup for deriving the transformation (A.3). The wedge bounded by $\tilde{r}=0$ and $\tilde{r}=\infty$ is near-NHEK. The wedge bounded by $r=0$ and $r=\infty$ is also near-NHEK. The two near-NHEK wedges are related to each other by a global time translation $\tau \rightarrow \tau-\pi / 2$.

Open Access. This article is distributed under the terms of the Creative Commons Attribution License (CC-BY 4.0), which permits any use, distribution and reproduction in any medium, provided the original author(s) and source are credited.

\section{References}

[1] Virgo and LigO Scientific collaborations, B.P. Abbott et al., Observation of gravitational waves from a binary black hole merger, Phys. Rev. Lett. 116 (2016) 061102 [arXiv: 1602.03837] [INSPIRE].

[2] Laser Interferometer Gravitational-Wave Observatory (LIGO) webpage, http://www.ligo.org.

[3] Evolved Laser Interferometer Space Antenna (eLISA) webpage, http://www.elisascience.org.

[4] C.S. Reynolds, Measuring black hole spin using X-ray reflection spectroscopy, Space Sci. Rev. 183 (2014) 277 [arXiv:1302.3260] [INSPIRE].

[5] L. Brenneman, Measuring supermassive black hole spins in active galactic nuclei, arXiv:1309.6334 [INSPIRE].

[6] J.M. Bardeen and G.T. Horowitz, The extreme Kerr throat geometry: a vacuum analog of $A d S_{2} \times S^{2}$, Phys. Rev. D 60 (1999) 104030 [hep-th/9905099] [INSPIRE].

[7] M. Guica, T. Hartman, W. Song and A. Strominger, The Kerr/CFT correspondence, Phys. Rev. D 80 (2009) 124008 [arXiv:0809.4266] [InSPIRE].

[8] A.P. Porfyriadis and A. Strominger, Gravity waves from the Kerr/CFT correspondence, Phys. Rev. D 90 (2014) 044038 [arXiv:1401.3746] [INSPIRE]. 
[9] S. Hadar, A.P. Porfyriadis and A. Strominger, Gravity waves from extreme-mass-ratio plunges into Kerr black holes, Phys. Rev. D 90 (2014) 064045 [arXiv:1403.2797] [INSPIRE].

[10] S. Hadar, A.P. Porfyriadis and A. Strominger, Fast plunges into Kerr black holes, JHEP 07 (2015) 078 [arXiv: 1504.07650] [INSPIRE].

[11] S.E. Gralla, A.P. Porfyriadis and N. Warburton, Particle on the innermost stable circular orbit of a rapidly spinning black hole, Phys. Rev. D 92 (2015) 064029 [arXiv:1506.08496] [INSPIRE].

[12] S.E. Gralla, S.A. Hughes and N. Warburton, Inspiral into Gargantua, Class. Quant. Grav. 33 (2016) 155002 [arXiv: 1603.01221] [INSPIRE].

[13] A. Lupsasca, M.J. Rodriguez and A. Strominger, Force-free electrodynamics around extreme Kerr black holes, JHEP 12 (2014) 185 [arXiv:1406.4133] [INSPIRE].

[14] F. Zhang, H. Yang and L. Lehner, Towards an understanding of the force-free magnetosphere of rapidly spinning black holes, Phys. Rev. D 90 (2014) 124009 [arXiv:1409.0345] [INSPIRE].

[15] A. Lupsasca and M.J. Rodriguez, Exact solutions for extreme black hole magnetospheres, JHEP 07 (2015) 090 [arXiv: 1412.4124] [INSPIRE].

[16] G. Compère and R. Oliveri, Near-horizon extreme Kerr magnetospheres, Phys. Rev. D 93 (2016) 024035 [Erratum ibid. D 93 (2016) 069906] [arXiv:1509.07637] [INSPIRE].

[17] S.E. Gralla, A. Lupsasca and A. Strominger, Near-horizon Kerr magnetosphere, Phys. Rev. D 93 (2016) 104041 [arXiv: 1602.01833] [InSPIRE].

[18] A.P. Porfyriadis, Y. Shi and A. Strominger, Photon emission near extreme Kerr black holes, arXiv: 1607.06028 [INSPIRE].

[19] M. Casals, S.E. Gralla and P. Zimmerman, Horizon instability of extremal Kerr black holes: nonaxisymmetric modes and enhanced growth rate, Phys. Rev. D 94 (2016) 064003 [arXiv: 1606.08505] [INSPIRE].

[20] S.E. Gralla, A. Zimmerman and P. Zimmerman, Transient instability of rapidly rotating black holes, Phys. Rev. D 94 (2016) 084017 [arXiv: 1608.04739] [INSPIRE].

[21] T. Jacobson and T.P. Sotiriou, Over-spinning a black hole with a test body, Phys. Rev. Lett. 103 (2009) 141101 [Erratum ibid. 103 (2009) 209903] [arXiv:0907.4146] [INSPIRE].

[22] M. Colleoni and L. Barack, Overspinning a Kerr black hole: the effect of self-force, Phys. Rev. D 91 (2015) 104024 [arXiv:1501.07330] [INSPIRE].

[23] M. Colleoni, L. Barack, A.G. Shah and M. van de Meent, Self-force as a cosmic censor in the Kerr overspinning problem, Phys. Rev. D 92 (2015) 084044 [arXiv: 1508.04031] [INSPIRE].

[24] I. Bredberg, T. Hartman, W. Song and A. Strominger, Black hole superradiance from Kerr/CFT, JHEP 04 (2010) 019 [arXiv:0907.3477] [INSPIRE].

[25] C. Merlin, A. Ori, L. Barack, A. Pound and M. van de Meent, Completion of metric reconstruction for a particle orbiting a Kerr black hole, Phys. Rev. D 94 (2016) 104066 [arXiv: 1609.01227] [INSPIRE].

[26] S.L. Detweiler and B.F. Whiting, Selfforce via a Green's function decomposition, Phys. Rev. D 67 (2003) 024025 [gr-qc/0202086] [inSPIRE]. 